ARTICLE

\title{
Peginterferon Lambda-1a for treatment of outpatients with uncomplicated COVID-19: a randomized placebo-controlled trial
}

Prasanna Jagannathan (10 1,2凶, Jason R. Andrews ${ }^{1,11}$, Hector Bonilla1,11, Haley Hedlin (D) 3,11, Karen B. Jacobson?', Vidhya Balasubramanian, ${ }^{3}$, Natasha Purington ${ }^{3}$, Savita Kamble ${ }^{4}$, Christiaan R. de Vries (1) ${ }^{1}$, Orlando Quintero ${ }^{1}$, Kent Feng (10 4, Catherine Ley ${ }^{1}$, Dean Winslow ${ }^{1}$, Jennifer Newberry ${ }^{5}$, Karlie Edwards ${ }^{1}$, Colin Hislop ${ }^{6}$, Ingrid Choong6, Yvonne Maldonado7, Jeffrey Glenn ${ }^{1,2}$, Ami Bhatt (1) 1,8, Catherine Blish (D) 1, Taia Wang (1) 1,2, Chaitan Khosla ${ }^{9}$, Benjamin A. Pinsky (10) ${ }^{1,10}$, Manisha Desai ${ }^{3}$, Julie Parsonnet $\mathbb{C}^{1}$ \& Upinder Singh ${ }^{1,2 \otimes}$

Type III interferons have been touted as promising therapeutics in outpatients with coronavirus disease 2019 (COVID-19). We conducted a randomized, single-blind, placebocontrolled trial (NCTO4331899) in 120 outpatients with mild to moderate COVID-19 to determine whether a single, $180 \mathrm{mcg}$ subcutaneous dose of Peginterferon Lambda-1a (Lambda) within 72 hours of diagnosis could shorten the duration of viral shedding (primary endpoint) or symptoms (secondary endpoint). In both the 60 patients receiving Lambda and 60 receiving placebo, the median time to cessation of viral shedding was 7 days (hazard ratio $[\mathrm{HR}]=0.81 ; 95 \%$ confidence interval $[\mathrm{Cl}] 0.56$ to 1.19). Symptoms resolved in 8 and 9 days in Lambda and placebo, respectively, and symptom duration did not differ significantly between groups (HR 0.94; $95 \% \mathrm{Cl} 0.64$ to 1.39 ). Both Lambda and placebo were welltolerated, though liver transaminase elevations were more common in the Lambda vs. placebo arm (15/60 vs 5/60; $p=0.027)$. In this study, a single dose of subcutaneous Peginterferon Lambda-1a neither shortened the duration of SARS-CoV-2 viral shedding nor improved symptoms in outpatients with uncomplicated COVID-19.

\footnotetext{
${ }^{1}$ Department of Medicine, Stanford University, Stanford, CA, USA. ${ }^{2}$ Department of Microbiology and Immunology, Stanford University, Stanford, CA, USA

${ }^{3}$ Quantitative Sciences Unit, Stanford University, Stanford, CA, USA. ${ }^{4}$ Stanford Center for Clinical Research, Stanford University, Stanford, CA, USA.

${ }^{5}$ Department of Emergency Medicine, Stanford University, Stanford, CA, USA. ${ }^{6}$ Department of Pediatrics, Stanford University, Stanford, CA, USA. ${ }^{7}$ Eiger BioPharmaceuticals, Palo Alto, CA, USA. ${ }^{8}$ Department of Genetics, Stanford University, Stanford, CA, USA. ${ }^{9}$ ChEM-H, Stanford University, Stanford, CA, USA. ${ }^{10}$ Department of Pathology, Stanford University, Stanford, CA, USA. ${ }^{11}$ These authors contributed equally: Jason R. Andrews, Hector Bonilla, Haley

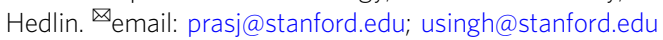


C oronavirus Disease 2019 (COVID-19), caused by the Severe Acute Respiratory Syndrome Coronavirus 2 (SARS-CoV-2), has led to more than 2 million deaths worldwide as of March 2021 ${ }^{1}$. Although most infected patients display mild symptoms, even uncomplicated infections can contribute to transmission to those with co-morbid conditions and other high risk groups, increasing overall mortality ${ }^{2}$. With the unprecedented health and economic threats imposed by COVID19 , therapeutics are urgently needed to shorten the duration of viral shedding, relieve symptoms, and prevent hospitalizations.

Interferons (IFNs) are promising anti-SARS-CoV-2 therapeutics, given their importance in the early response to viral infections ${ }^{3}$. Innate immune sensing of viral nucleic acids leads to production of type I (IFN- $\alpha$, IFN- $\beta$ ) and type III (IFN- $\lambda$ ) IFNs that, after binding to their cognate receptors, activate genes critical for host protection ${ }^{4-6}$. SARS-CoV-2 encodes proteins that suppress production of endogenous IFN ${ }^{7}$, and infection has been associated with markedly reduced type I and type III IFN signaling ${ }^{8}$, particularly in patients with severe manifestations of disease $^{9,10}$. Both type I and type III IFNs inhibit SARS-CoV-2 in vitro $^{11,12}$, suggesting potential utility of exogenous IFN administration to aid in viral control and prevent disease progression. In support of this hypothesis, recent trials in hospitalized COVID-19 patients have reported that both subcutaneous and inhaled type I IFN administration may reduce the duration of viral shedding and symptoms ${ }^{13-17}$.

Whereas cognate receptors to type I IFNs are expressed ubiquitously, the receptor complex (IL28R) for IFN- $\lambda$ is expressed on only a few cell types, including epithelial cells in the gastrointestinal and respiratory tracts $4,18,19$. These cellular affinities have led investigators to use this agent to target viral hepatitides ${ }^{20,21}$ and respiratory viral infections ${ }^{18}$. In a murine model of influenza infection, IFN- $\lambda$ treatment post-infection was associated with significantly lower mortality compared to mice treated with IFN- $\alpha$, and this was associated with lower influenza viral loads ${ }^{22}$. A pegylated form of recombinant IFN- $\lambda$, Peginterferon Lambda-1a (Lambda) has been developed for the treatment of chronic viral hepatitis. Lambda, given weekly as $180 \mathrm{mcg}$ subcutaneous injections, has comparable antiviral efficacy and an improved tolerability profile compared with type I IFN for the treatment of hepatitis ${ }^{23}$, likely due to its relatively limited receptor distribution. In a murine model of SARS-CoV-2 infection, subcutaneous administration of Lambda prophylactically or early after infection diminished SARS-CoV-2 replication in the lower respiratory tracts of mice in vivo ${ }^{11}$.

Lambda has thus emerged as a promising treatment candidate for SARS-CoV-2 24,25 given a plausible mechanism of action, the suppression of IFN activity by SARS-CoV-2, and in vitro and in vivo studies showing that IFN- $\lambda$ administration can inhibit SARS-CoV-2 replication. To date, no therapies have been approved for outpatients with mild to moderate COVID-19 disease. We therefore conducted a randomized, placebo-controlled trial of Lambda for outpatients with uncomplicated SARS-CoV-2 infection. We tested the hypothesis that a single, $180 \mathrm{mcg}$ subcutaneous injection of Lambda would be associated with a shorter duration of viral shedding in comparison to a normal saline placebo injection.

In this work, we show that a single subcutaneous injection of Lambda in outpatients with uncomplicated SARS-CoV-2 infection did not significantly reduce time to viral clearance or resolution of symptoms compared with placebo. Lambda was welltolerated, with few adverse effects, though asymptomatic liver transaminase elevations occurred more frequently in participants randomized to Lambda compared with placebo.

\section{Results}

We enrolled 120 participants between April 25 and July 17, 2020, of whom $110(91.7 \%)$ completed 28 days of follow up (Fig. 1a).

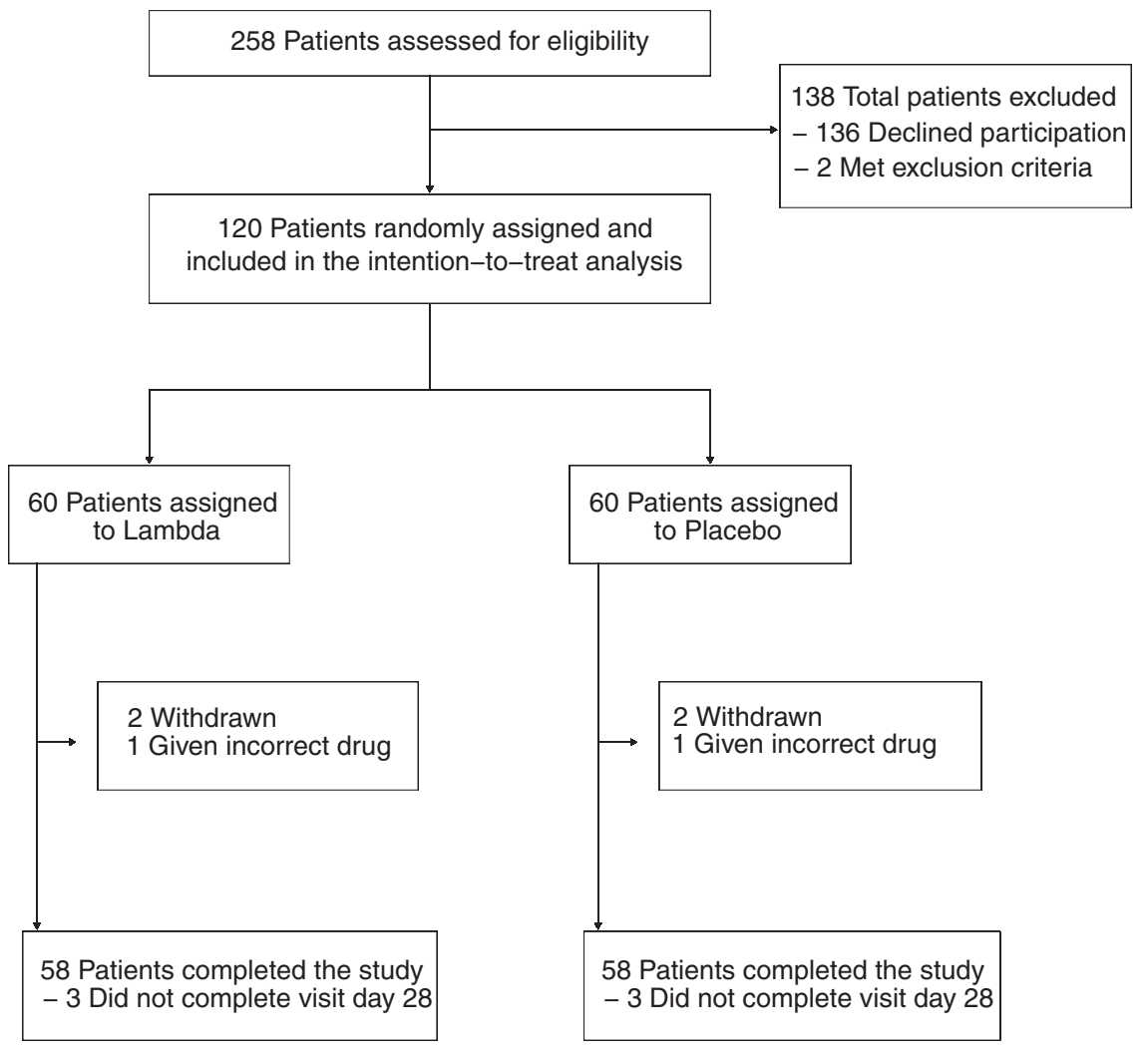

Fig. 1 CONSORT diagram. Trial schematic showing participants screened, randomized, and followed through study completion. 
Table 1 Baseline characteristics of study participants.

\begin{tabular}{|c|c|c|c|}
\hline & \multicolumn{3}{|c|}{ Treatment arm } \\
\hline & $\begin{array}{l}\text { Lambda } \\
(n=60)\end{array}$ & $\begin{array}{l}\text { Placebo } \\
(n=60)\end{array}$ & $\begin{array}{l}\text { Overall } \\
(n=120)\end{array}$ \\
\hline $\begin{array}{l}\text { Age in years, median } \\
\text { (range) }\end{array}$ & $37(18-66)$ & $34(20-71)$ & $36(18-71)$ \\
\hline Female, $n(\%)$ & $24(40.0 \%)$ & $26(43.3 \%)$ & $50(41.7 \%)$ \\
\hline \multicolumn{4}{|l|}{ Race/Ethnicity, n (\%) } \\
\hline Latinx & $34(56.7 \%)$ & $41(68.3 \%)$ & $75(62.5 \%)$ \\
\hline White & $18(30.0 \%)$ & $15(25.0 \%)$ & $33(27.5 \%)$ \\
\hline Asian & $3(5.0 \%)$ & $4(6.7 \%)$ & $7(5.8 \%)$ \\
\hline $\begin{array}{l}\text { Native Hawaiian or } \\
\text { other Pacific Islander }\end{array}$ & $2(3.3 \%)$ & $0(0 \%)$ & $2(1.7 \%)$ \\
\hline Unknown & $2(3.3 \%)$ & $0(0 \%)$ & $2(1.7 \%)$ \\
\hline More than one race & $1(1.7 \%)$ & $0(0 \%)$ & $1(0.8 \%)$ \\
\hline BMI $\left(\mathrm{kg} / \mathrm{m}^{2}\right)$, & 27.6 & 28.5 & 27.7 \\
\hline median (IQR) & $(25.4-31.1)$ & $(24.8-32.3)$ & $(24.9-32.0)$ \\
\hline \multicolumn{4}{|l|}{ Comorbid conditions } \\
\hline Hypertension & $9(15.0)$ & $5(8.3)$ & $14(11.7)$ \\
\hline Diabetes & $4(6.7)$ & $8(13.3)$ & $12(10.0)$ \\
\hline Asthma & $2(3.3)$ & $2(3.3)$ & $4(3.3)$ \\
\hline Heart disease & $3(5.0)$ & $1(1.7)$ & $4(3.3)$ \\
\hline \multicolumn{4}{|c|}{ Concomitant medications } \\
\hline Antihypertensive & $9(15.0)$ & $5(8.3)$ & $14(11.7)$ \\
\hline Oral hypoglycemic & $4(6.7)$ & $8(13.3)$ & $12(10.0)$ \\
\hline Insulin & $0(0.0)$ & $1(1.7)$ & $1(0.8)$ \\
\hline Aspirin & $1(1.7)$ & $2(3.3)$ & $3(2.5)$ \\
\hline Statin & $3(5.0)$ & $5(8.3)$ & $8(6.7)$ \\
\hline Albuterol & $2(3.3)$ & $0(0.0)$ & $2(1.7)$ \\
\hline $\begin{array}{l}\text { Asymptomatic at } \\
\text { baseline, } n(\%)\end{array}$ & $6(10.0 \%)$ & $2(3.3 \%)$ & $8(6.7 \%)$ \\
\hline $\begin{array}{l}\text { Duration of symptoms in } \\
\text { days prior to } \\
\text { randomization, median } \\
(\text { IQR) a }\end{array}$ & $4(3-6)$ & $5(3-5)$ & $5(3-6)$ \\
\hline \multicolumn{4}{|c|}{ Symptoms at baseline, $n(\%)$} \\
\hline Fatigue & $33(55 \%)$ & $42(70 \%)$ & $75(62.5 \%)$ \\
\hline Cough & $33(55.0 \%)$ & $36(60.0 \%)$ & $69(57.5 \%)$ \\
\hline Headache & $29(48.3 \%)$ & $36(60 \%)$ & $65(54.2 \%)$ \\
\hline Myalgias & $29(48.3 \%)$ & $34(56.7 \%)$ & $63(52.5 \%)$ \\
\hline $\begin{array}{l}\text { Decreased taste } \\
\text { or smell }\end{array}$ & $25(41.7 \%)$ & $32(53.3 \%)$ & $57(47.5 \%)$ \\
\hline Chills & $22(36.7 \%)$ & $27(45 \%)$ & $49(40.8 \%)$ \\
\hline Sore throat & $22(36.7 \%)$ & $23(38.3 \%)$ & $45(37.5 \%)$ \\
\hline Joint pain & $19(31.7 \%)$ & $19(31.7 \%)$ & $38(31.7 \%)$ \\
\hline Diarrhea & $16(26.7 \%)$ & $18(30 \%)$ & $34(28.3 \%)$ \\
\hline Nausea & $11(18.3 \%)$ & $23(38.3 \%)$ & $34(28.3 \%)$ \\
\hline Shortness of breath & $16(26.7 \%)$ & $16(26.7 \%)$ & $32(26.7 \%)$ \\
\hline Chest pain/pressure & $14(23.3 \%)$ & $13(21.7 \%)$ & $27(22.5 \%)$ \\
\hline Runny nose & $10(16.7 \%)$ & $16(26.7 \%)$ & $26(21.7 \%)$ \\
\hline Abdominal pain & $7(11.7 \%)$ & $7(11.7 \%)$ & $14(11.7 \%)$ \\
\hline Rash & $4(6.7 \%)$ & $5(8.3 \%)$ & $9(7.5 \%)$ \\
\hline Vomiting & $1(1.7 \%)$ & $5(8.3 \%)$ & $6(5 \%)$ \\
\hline \multicolumn{4}{|l|}{ Vital signs at enrollment } \\
\hline $\begin{array}{l}\text { Temperature } 99.5 \mathrm{~F} \\
+, \mathrm{n}(\%)\end{array}$ & $6(10.0 \%)$ & $7(11.7 \%)$ & $13(10.8 \%)$ \\
\hline $\begin{array}{l}\text { Oxygen saturation, } \\
\text { median (IQR) }\end{array}$ & $98(2.5)$ & $99(3)$ & $98(3)$ \\
\hline \multicolumn{4}{|c|}{ Baseline laboratory values, median (IQR) } \\
\hline $\begin{array}{l}\text { White blood cell } \\
\text { (WBC) count, cells } / \mu l\end{array}$ & $5.5(4.3-6.8)$ & $\begin{array}{l}5.6 \\
(4.0-7.5)\end{array}$ & $5.5(4.1-7.1)$ \\
\hline $\begin{array}{l}\text { Absolute lymphocyte } \\
\text { count (ALC), cells } / \mu \text { l }\end{array}$ & $1.5(1.2-1.9)$ & $1.5(1.2-2.3)$ & $1.5(1.2-2.2)$ \\
\hline $\begin{array}{l}\text { Aspartate } \\
\text { aminotransferase, IU/L }\end{array}$ & $31(26-41)$ & $\begin{array}{l}30 \\
(25-39.3)\end{array}$ & $30(25-41)$ \\
\hline $\begin{array}{l}\text { Alanine } \\
\text { aminotransferase, IU/L }\end{array}$ & $\begin{array}{l}32.5 \\
(21-52.3)\end{array}$ & $\begin{array}{l}30.5 \\
(23-47.5)\end{array}$ & $\begin{array}{l}31.5 \\
(22-50.3)\end{array}$ \\
\hline
\end{tabular}

Table 1 (continued)

\begin{tabular}{|c|c|c|c|}
\hline & \multicolumn{3}{|l|}{ Treatment arm } \\
\hline & $\begin{array}{l}\text { Lambda } \\
(n=60)\end{array}$ & $\begin{array}{l}\text { Placebo } \\
(n=60)\end{array}$ & $\begin{array}{l}\text { Overall } \\
(n=120)\end{array}$ \\
\hline Baseline oropharyngeal & 30.9 & 29.3 & 30.3 \\
\hline $\begin{array}{l}\text { SARS-CoV-2 cycle } \\
\text { threshold, median }(\mathrm{IQR})^{\mathrm{b}}\end{array}$ & $(26.4-33.8)$ & $(26.4-34.3)$ & $(26.4-34.3)$ \\
\hline 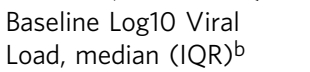 & $4.2(3.3-5.5)$ & $4.7(3.2-5.5)$ & $4.4(3.2-5.5)$ \\
\hline $\begin{array}{l}\text { Baseline SARS-CoV-2 lgG } \\
\text { seropositivity, } n(\%)\end{array}$ & $21(35.0 \%)$ & $28(46.7 \%)$ & $49(40.8 \%)$ \\
\hline $\begin{array}{l}\text { Sum of risk factors, } \\
\text { median (IQR) }\end{array}$ & $3(2-3)$ & $3(2-4)$ & $3(2-3)$ \\
\hline \multicolumn{4}{|c|}{$\begin{array}{l}\text { Sum of risk factors is defined as the number of relevant severe disease risk factors present at } \\
\text { baseline (presence of either temperature of } 99.5 \mathrm{~F}+\text {, cough, or shortness of breath; age } 60+; \\
\text { male sex; Black race; Latinx ethnicity; BMI 30+; ALC }<1000 ; A L T 94+\text { ). Source data available at } \\
\text { https://purl.stanford.edu/hc972ys6733. } \\
\text { IQR inner quartile range, ASD absolute standardized difference. } \\
\text { aAmong } n=103 \text { participant who reported symptoms prior to randomization ( } n=48 \text { in lambda } \\
\text { and } n=55 \text { in placebo). } \\
\text { bAmong } n=87 \text { participants with detectable OP virus ( } n=44 \text { in lambda and } n=43 \text { in placebo). }\end{array}$} \\
\hline
\end{tabular}

The median age was 36 years (range 18-71), 50 participants (41.7\%) were female, and $75(62.5 \%)$ were Latinx ethnicity (Table 1). Eight (6.7\%) participants were asymptomatic at baseline. Of those with symptoms, the median duration of symptoms prior to randomization was 5 days. The most common symptoms were fatigue, cough, headache, and myalgias (Table 1, Supplementary Fig. 1). Only 13 (10.8\%) participants had an elevated temperature $\left(>99.5^{\circ} \mathrm{F}\right)$ at baseline; the median oxygen saturation was $98 \%$. The proportion of missing follow-up visits was $44 / 960$ (4.6\%). Only 16/960 visits were missed among patients not hospitalized or prematurely withdrawn.

The presence of SARS-CoV-2 RNA was assessed at baseline and at 8 follow-up visits using oropharyngeal swabs, a Centers for Disease Control approved method for SARS-CoV-2 detection ${ }^{26}$. This method was selected given the frequency of repeated assessments and improved tolerability for participants compared with nasopharyngeal swabs. The median SARS-CoV-2 oropharyngeal viral PCR cycle threshold at enrollment was 30.3 (corresponding to a median viral load of $4.4 \log 10$ copies $/ \mathrm{ml}$ ), and was comparable between groups (Table 1). Patients were randomized within a median of 2 days (range $0-3$ days) following diagnosis. The median time from onset of symptoms before diagnosis was 5 days (IQR 3-6).

IgG antibody titers against the SARS-CoV-2 spike receptorbinding domain (RBD) were assessed at enrollment by enzymelinked immunosorbent assay ${ }^{27}$. At enrollment, 49 (40.8\%) participants were SARS-CoV-2 IgG seropositive. Baseline SARSCoV-2 serostatus did not significantly differ between groups (Table 1). The median duration of symptoms prior to enrollment was shorter in SARS-CoV-2 IgG seronegative vs. seropositive participants (median [interquartile range [IQR]]: 3.5 [2.5-5] days vs. 5 [4-7] days, $P=0.0051$ ). Seronegative participants also had significantly higher oropharyngeal viral RNA levels at enrollment compared with seropositive participants (median [IQR] $\log _{10}$ viral load 4.4 [2.5] vs. $2.0[2.4]$ ).

Of 120 enrolled participants, 60 were randomized to receive Lambda and 60 randomized to receive placebo and included in the analysis. The median time to cessation of oropharyngeal viral shedding was 7 days in both arms (95\% CI 5-10 days for placebo vs. 5-13 days for Lambda, Table 2). There was no significant difference in the adjusted hazard ratio (aHR) for shedding cessation between Lambda and placebo; participants in the Lambda arm were $19 \%$ less likely to cease shedding at any point during the study period compared to participants in the placebo arm 
Table 2 Efficacy and safety outcomes.

\begin{tabular}{|c|c|c|c|c|}
\hline & \multicolumn{2}{|l|}{ Treatment arm } & \multicolumn{2}{|l|}{ Measure of association } \\
\hline & Lambda $(n=60)$ & Placebo $(n=60)$ & aHR $(95 \% \mathrm{Cl})$ & $p$-value \\
\hline \multicolumn{5}{|l|}{ Primary efficacy outcome } \\
\hline $\begin{array}{l}\text { Duration until viral shedding cessation in days, median }(95 \% \mathrm{Cl}) \\
\text { Secondary efficacy outcomes }\end{array}$ & $7(5-13)$ & $7(5-10)$ & $0.81(0.56,1.19)$ & 0.29 \\
\hline Duration until resolution of symptoms in days, median $(95 \% \mathrm{Cl})$ & $8(6-11)$ & $9(5-11)$ & $0.94(0.64,1.39)$ & 0.76 \\
\hline Hospitalizations by Day $28, n$ participants (\%) & $2(3.3 \%)$ & $2(3.3 \%)$ & - & 1 \\
\hline Emergency Department visits by Day $28, n$ participants (\%) & $5(8.3 \%)$ & $3(5 \%)$ & $\bar{\Delta}$ Log change $(95 \% \mathrm{Cl})$ & $\begin{array}{l}0.71 \\
p \text {-value }\end{array}$ \\
\hline Log Oropharyngeal viral load over time, mean change at day 14 (SD) & $-4.3(4.3)$ & $-4.9(4.7)$ & $\begin{array}{l}-0.06(-1.23,1.11) \\
\Delta \text { AUC }(95 \% \mathbf{C l})\end{array}$ & 0.91 \\
\hline $\begin{array}{l}\log _{10} \text { viral load area under the curve through day } 14 \text {, median (IQR) } \\
\text { Exploratory efficacy outcomes }\end{array}$ & $28.5(20.1)$ & $29.6(19.0)$ & $\begin{array}{l}1.01(0.85,1.16) \\
\text { aHR }(95 \% \mathbf{C l})\end{array}$ & $\begin{array}{l}0.95 \\
p \text {-value }\end{array}$ \\
\hline Duration until sustained symptom resolution in days, median $(95 \% \mathrm{Cl})$ & $20(16-27)$ & $20(17-24)$ & $0.92(0.60,1.41)$ & 0.70 \\
\hline Duration until respiratory symptom resolution in days, median $(95 \% \mathrm{Cl})$ & $6(4-7)$ & $4(2-7)$ & $0.99(0.64,1.53)$ & 0.95 \\
\hline $\begin{array}{l}\text { Duration until systemic and respiratory symptom resolution in days, } \\
\text { median }(95 \% \mathrm{Cl})\end{array}$ & $8(6-11)$ & $5.5(5-10)$ & $0.93(0.63,1.38)$ & 0.73 \\
\hline $\begin{array}{l}\text { Duration until disease progression in days, median among those who } \\
\text { progress }^{\star}(\mathrm{IQR})\end{array}$ & $5(1)$ & $2(1)$ & $1.38(0.52,3.63)$ & 0.52 \\
\hline \multicolumn{5}{|l|}{ Safety outcomes } \\
\hline Serious adverse events, $n(\%)$ & $2(3.3 \%)$ & $2(3.3 \%)$ & - & 1 \\
\hline Number of adverse events, $n$ & 36 & 30 & - & \\
\hline Participants with adverse events, $n$ (\%) & $25(41.7 \%)$ & $21(35.0 \%)$ & - & 0.57 \\
\hline LFT-related adverse events, $n$ & 16 & 5 & - & \\
\hline Participants with LFT-related adverse events, $n(\%)$ & $15(25.0 \%)$ & $5(8.3 \%)$ & - & 0.027 \\
\hline
\end{tabular}

(aHR 0.81, 95\% confidence interval [CI] 0.56- 1.19; $p=0.29$, Fig. 2a). Overall, 108 participants met the primary endpoint and were not censored. Because two participants, after randomization, inadvertently were injected with the incorrect syringe, we also conducted an as-treated analysis according to treatment actually received. Findings from an as-treated analysis (aHR 0.83, 95\% CI $0.56-1.21 ; p=0.33$ ) and an analysis performed in symptomatic patients only (aHR $0.77,95 \%$ CI $0.52-1.15, p=0.21$ ) were similar.

No significant difference in time to resolution of symptoms (aHR $0.94 ; 95 \%$ CI $0.64-1.39 ; p=0.76$, Fig. 2b) or sustained resolution of symptoms (aHR 0.92; 95\% CI 0.60-1.41; $p=0.70$ ) was observed, nor did we find any significant difference in resolution of symptom complexes (Table 2, Supplementary Fig. 2). Overall, 17 participants had evidence of disease progression, defined as hospitalization, presentation to the emergency department, or worsening cough or shortness of breath defined as an increase in severity of two points or more on a five-point scale. Time to clinical progression was not significantly different between the two arms (aHR 1.38; 95\% CI 0.52-3.63; $p=0.52$, Table 2). At-home oxygen saturation measurements also did not differ significantly between groups (Supplementary Fig. 3).

Trajectory of viral RNA levels did not vary by treatment arm $(p=0.91$, Supplementary Fig. 4$)$ nor did viral RNA area under the curve $(p=0.95$, Table 2$)$. In exploratory analysis, seropositivity at baseline was associated with significantly hastened shedding cessation. The median time to shedding cessation was 10 days in SARS-CoV-2 IgG seronegative individuals vs. 3 days in seropositive individuals (95\% CI 7-14 days for seronegative vs. 1-6 days for seropositive, aHR 2.65, 95\% CI 1.74-4.03, $\mathrm{p}<0.001$, Supplementary Fig. 5). Baseline serostatus significantly modified the effect of treatment on time to shedding cessation $(p=0.03)$. Among seronegative individuals, point estimates suggest that Lambda delayed shedding cessation compared with placebo
(aHR 0.66, 95\% CI 0.39-1.10, Fig. 3a). Among seropositive individuals, point estimates suggest that Lambda hastened shedding cessation compared with placebo (aHR 1.58, 95\% CI $0.88-2.86$, Fig. 3a).

Higher oropharyngeal viral RNA levels at baseline were associated with significantly delayed shedding cessation (aHR 0.32 comparing baseline CT $<30$ vs. baseline CT $\geq 30$, 95\% CI $0.21-0.50, p<0.001$, Supplementary Fig. 6). Although baseline oropharyngeal viral RNA levels did not significantly modify the effect of treatment on time to shedding cessation $(p=0.15)$, among individuals with high baseline viral RNA levels, Lambda tended to delay shedding cessation compared with placebo (aHR $0.51,95 \%$ CI $0.26-1.04$, Fig. 3b). There was no difference in shedding cessation between arms with low baseline viral RNA levels (aHR 0.95, 95\% CI 0.60-1.52, Fig. 3b). No other baseline features of interest significantly modified the effect of treatment and the primary outcome (Table S1).

Twenty-five (42\%) Lambda and 21 (35\%) placebo participants experienced adverse events (Table 2). Two serious adverse events (hospitalizations) were reported in each arm, with all four hospitalizations due to hypoxia/worsening COVID-19. Liver transaminase elevations were more common in the Lambda vs. placebo arm (15 vs. 5; $p=0.027)$. Furthermore, we observed significant elevations in alanine transaminase levels from day 0 to day 5 among individuals randomized to Lambda, but not among individuals randomized to placebo (Supplementary Fig. 7). However, there were no associated symptoms and lab abnormalities were not sustained.

\section{Discussion}

In this study of outpatients with uncomplicated SARS-CoV-2 infection, a single subcutaneous injection of Lambda did not significantly reduce time to viral clearance or resolution of symptoms compared with placebo. We recruited participants 

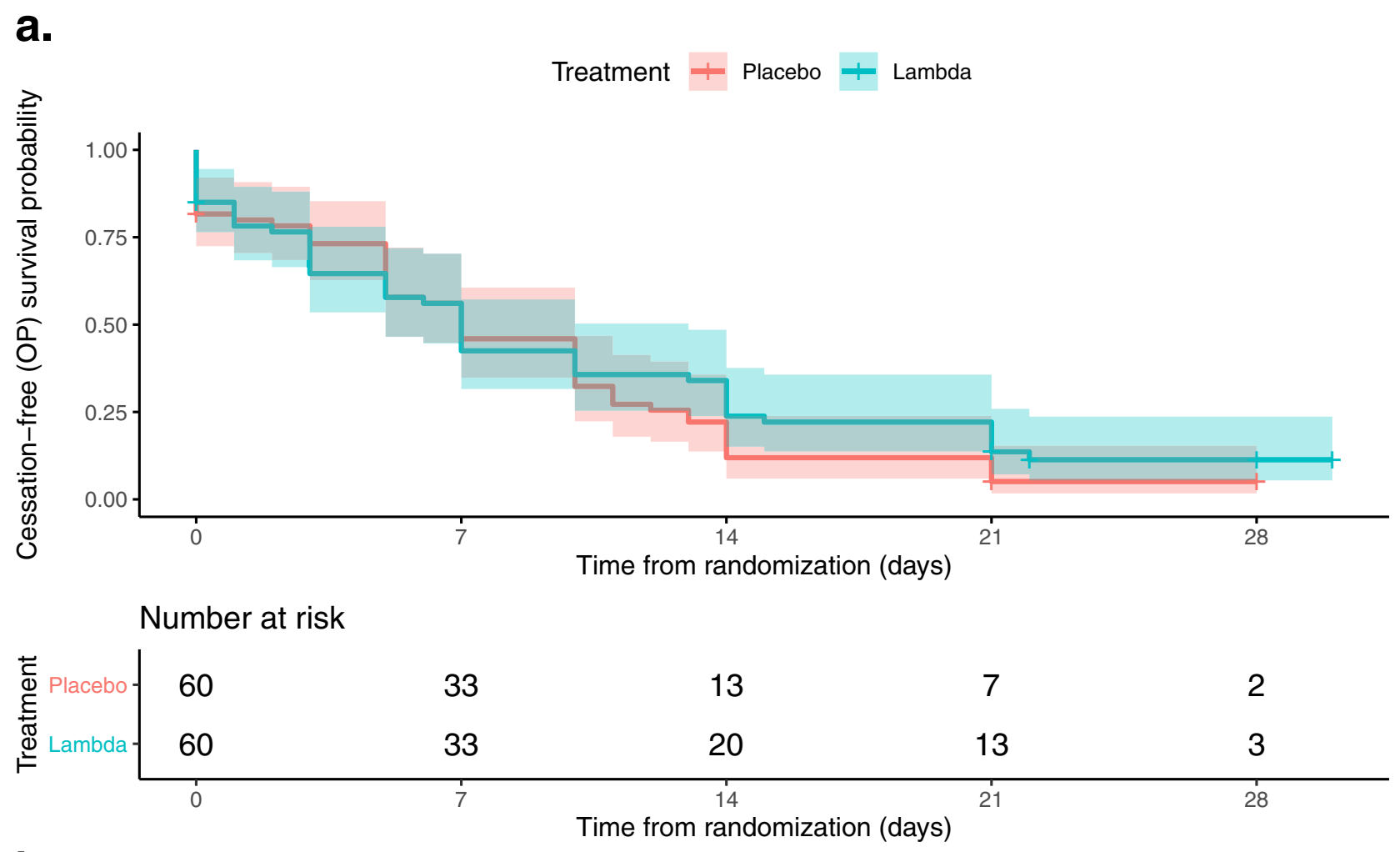

b.

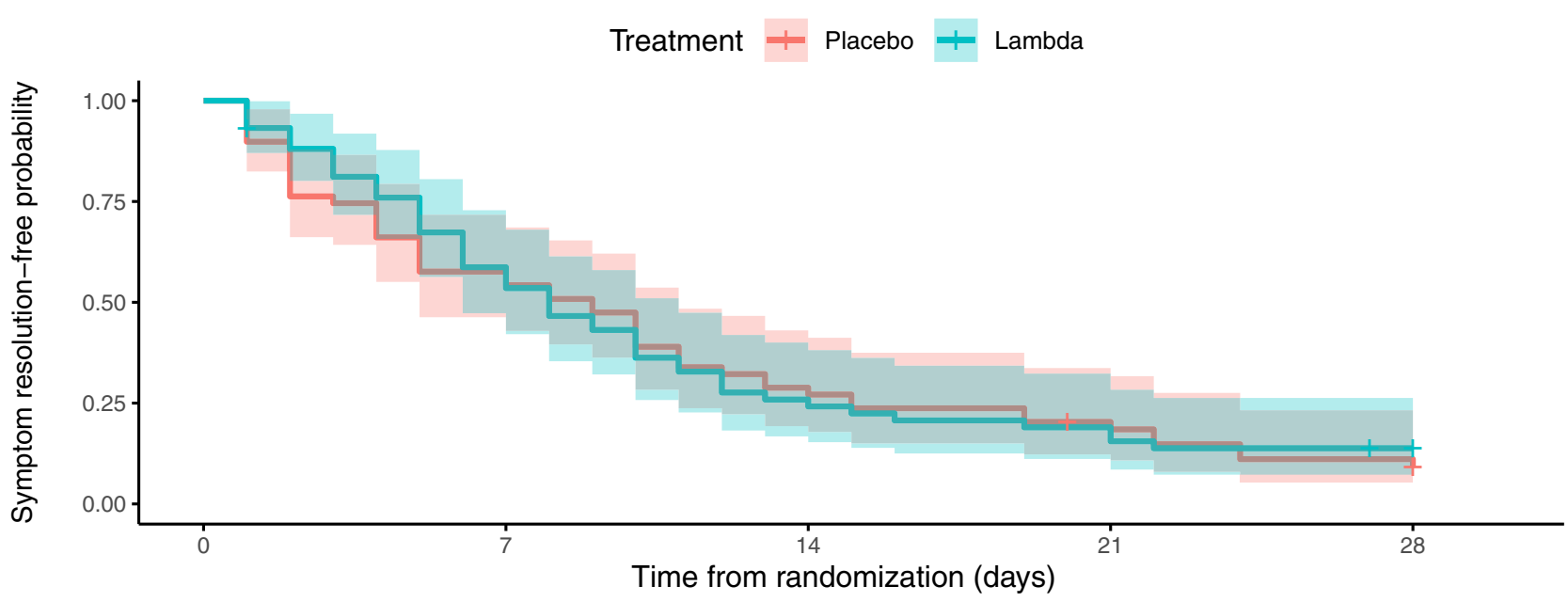

Number at risk

\begin{tabular}{|c|c|c|c|c|c|}
\hline 氙 Placebo- & 59 & 34 & 17 & 11 & 6 \\
\hline 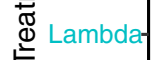 & 59 & 34 & 15 & 11 & 7 \\
\hline & 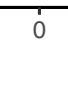 & 7 & $\begin{array}{l}14 \\
\text { and }\end{array}$ & 21 & 28 \\
\hline
\end{tabular}

Fig. 2 Kaplan-Meier analyses of the primary and key secondary outcome in the intention-to-treat population. a Time until cessation of SARS-CoV-2 viral shedding from oropharyngeal swabs stratified by treatment arm, Lambda (blue) vs. placebo (red). b Time until resolution of all symptoms stratified by treatment arm, Lambda (blue) vs. placebo (red). In both panels, solid lines represent Kaplan-Meier survival probability; shading represents $95 \%$ confidence intervals. Source data available at https://purl.stanford.edu/hc972ys6733.

within $72 \mathrm{~h}$ of diagnosis, giving us an excellent opportunity to intervene early within the course of infection. We attained excellent follow-up and retention, with few missed visits $(<5 \%)$, and little missing data. Despite these strengths and compelling preclinical data-i.e., a plausible mechanism of action, the suppression of IFN activity by respiratory coronaviruses, and both in vitro and in vivo studies showing inhibition of SARS$\mathrm{CoV}-2$ replication by IFN- $\lambda^{11,12}$-this phase 2 trial yielded little promise of efficacy at the tested dose and administration schedule. Lambda was well-tolerated, with few adverse effects, though 
a. Strata $+\underset{\text { seronegative }}{\text { Lambda }}+\underset{\text { seropositive }}{\text { Lambda }}+\underset{\text { Placebo }}{\text { seronegative }}+\underset{\text { Placebo }}{\text { seropositive }}$

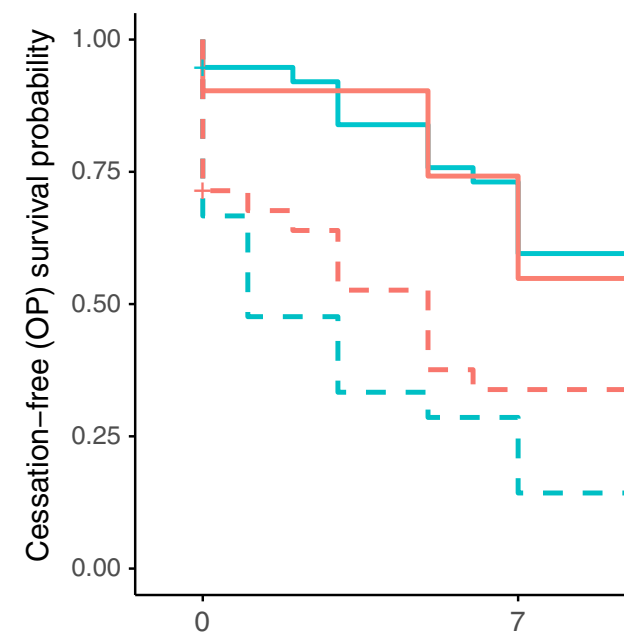

Number at risk

\begin{tabular}{|c|c|c|c|c|c|}
\hline \begin{tabular}{r|} 
Lambda \\
seronegative
\end{tabular} & 38 & 27 & 18 & 12 & 3 \\
\hline 㞼 $\begin{array}{r}\text { Lambda } \\
\text { seropositive }\end{array}$ & 21 & 6 & 2 & 1 & 0 \\
\hline $\begin{array}{l}\text { Placebo } \\
\text { ஸे } \\
\text { seronegative }\end{array}$ & 31 & 23 & 7 & 5 & 2 \\
\hline $\begin{array}{r}\text { Placebo } \\
\text { seropositive }\end{array}$ & 28 & 9 & 5 & 2 & 0 \\
\hline & 0 & 7 & 14 & 21 & 28 \\
\hline
\end{tabular}

b.

Time from randomization (days)

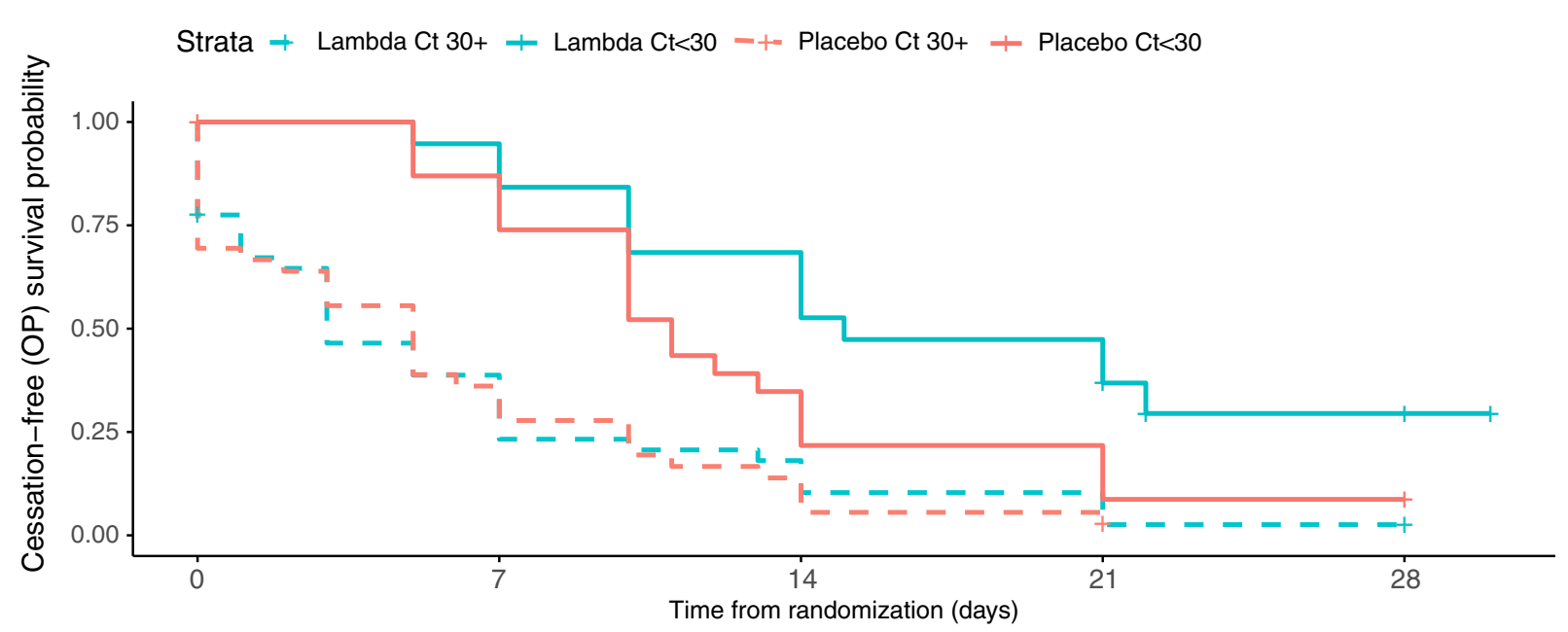

Number at risk

\begin{tabular}{|c|c|c|c|c|c|}
\hline Lambda Ct 30+. & 40 & 15 & 7 & 4 & 1 \\
\hline$\frac{\pi}{\pi}$ Lambda Ct<30. & 19 & 18 & 13 & 9 & 2 \\
\hline ڤे Placebo Ct 30+ & 36 & 13 & 5 & 2 & 0 \\
\hline Placebo $\mathrm{Ct}<30-$ & 24 & 20 & 8 & 5 & 2 \\
\hline & 0 & 7 & $\begin{array}{l}14 \\
\text { ran }\end{array}$ & 21 & 28 \\
\hline
\end{tabular}

Fig. 3 Kaplan-Meier analyses of the primary outcome, stratified by baseline seropositivity and viral load. a Time until cessation of SARS-CoV-2 viral shedding from oropharyngeal swabs stratified by baseline SARS-CoV-2 seropositivity, seropositive (dashed) and seronegative (solid), and treatment arm, Lambda (blue) vs. placebo (red). b Time until cessation of SARS-CoV-2 viral shedding from oropharyngeal swabs stratified by baseline SARS-CoV-2 oropharyngeal virus CT value, CT value $\geq 30$ (dashed) and CT value $<30$ (solid), and treatment arm, Lambda (blue) vs. placebo (red). In both panels, solid lines represent Kaplan-Meier survival probability; shading represents 95\% confidence intervals. Source data available at https://purl.stanford.edu/ hc972ys6733. 
asymptomatic liver transaminase elevations occurred more frequently in participants randomized to Lambda and are consistent with previous reports ${ }^{23,28}$.

The lack of effect of Lambda was surprising given recently described in vitro data and benefits seen in an in vivo model with early therapeutic and prophylactic administration ${ }^{12}$. There are several potential reasons for this lack of benefit. First, a single, $180 \mu \mathrm{g}$ subcutaneous injection of Lambda may not achieve adequate therapeutic levels of drug in the upper respiratory epithelia. In an in vitro model of SARS-CoV-2 infection, human epithelial cells pre-treated with $10 \mathrm{ng} / \mathrm{ml}$ of Lambda had a $\sim 1$ log reduction in viral titers. It is unclear whether these tissue levels are achievable, since, in Phase 1 and Phase 2 studies of single-dose subcutaneous administration of $180 \mu \mathrm{g}$ Lambda, $C_{\max }$ values ranged from 1.06 to $2.41 \mathrm{ng} / \mathrm{mL}$ (median $T_{\max }, 8-25 \mathrm{~h}$, range $1-120 \mathrm{~h}^{29}$.) Consistent with this, a murine model of SARS-CoV-2 infection found that subcutaneous administration of Lambda resulted in significant reductions of SARS-CoV-2 viral titers in lower, but not upper, respiratory epithelium ${ }^{12}$. The dose of Lambda used in this model was $2 \mu \mathrm{g}$ per mouse; the human equivalent dose (HED) is $8.1 \mu \mathrm{g} / \mathrm{kg} / \mathrm{dose}^{30}$. In an average $70 \mathrm{~kg}$ adult, $180 \mu \mathrm{g}$ Lambda would result in $2.6 \mu \mathrm{g} / \mathrm{kg} / \mathrm{dose}, 1 / 3$ of the dose given to mice. Though higher, or more frequent, dosing may have been beneficial, subcutaneous doses $>180 \mathrm{mcg}$ in humans are limited by increasing drug toxicity, including significant liver transaminase elevations ${ }^{23}$. Second, although we attempted to randomize participants as soon as possible after the COVID-19 diagnosis was made, the median symptom duration was 5 days at the time of randomization, and $40 \%$ of participants were already SARS-CoV-2 IgG positive at enrollment. It is possible that earlier administration, or prophylactic administration prior to established infection, would have been beneficial. Arguing against this, we observed no evidence of benefit among SARS-CoV-2 seronegative individuals, who presumably have been infected a shorter period of time. Finally, IFN- $\lambda$ has been shown to disrupt the lung epithelial barrier in mice, leading to worsened disease course and increase susceptibility to bacterial superinfection ${ }^{31,32}$. Furthermore, IFN- $\lambda$ has also been shown to inhibit influenza virus-stimulated $\mathrm{B}$-cell activation and antibody production ${ }^{33}$, suggesting negative impacts on the development of adaptive immune responses critical to resolution of infection. These may negate any positive antiviral effects.

Our results differ somewhat from those seen in a similar, but smaller, randomized controlled trial of Lambda at the same dose in COVID-19 outpatients in Toronto ${ }^{34}$. In the Toronto study, Feld and colleagues randomized 60 outpatients to a single subcutaneous injection of $180 \mu \mathrm{g}$ Lambda $(n=30)$ or placebo $(n=$ 30) within 7 days of symptom onset or first swab if asymptomatic. The primary outcome was proportion negative for SARS-CoV-2 RNA by self-collected mid turbinate swabs on day 7 post injection. The authors found that, overall, 24/30 (80\%) individuals in the Lambda arm, vs. $19 / 30$ (63\%) in the placebo arm, were negative for SARS-CoV-2 RNA on day $7(p=0.15)$. However, baseline mid-turbinate viral loads were higher in the Lambda arm (6.16 $\log 10$ copies/ml) vs. the placebo arm $(4.87 \log 10$ copies $/ \mathrm{ml})$ among those with detectable viral loads at enrollment. After adjusting for baseline viral load, patients in the Lambda group were more likely to be clear of virus by Day 7 after dosing (OR $4.1295 \%$ CI $1.216-7, p=0.029)$. Among individuals with midturbinate viral loads of $>10^{6}$ copies/ml at enrollment (19 in the lambda arm vs. 16 in placebo), a greater proportion of Lambda participants were negative at day 7 than those in placebo $(15 / 19$ $(79 \%)$ in Lambda vs. 6/16 (38\%) in placebo, $p=0.01)$; no significant difference was observed among individuals with viral loads of $<10^{6}$ copies/ml at enrollment. While these secondary analyses adjusting for baseline viral load were pre-specified, they were not the primary outcome. Thus, given the relatively small sample size among those with higher viral loads $(n=35)$, the corresponding type I error for this particular result is unclear. In contrast to this finding, despite a similar duration of viral shedding among higher viral load subjects, in our study, Lambda appeared to prolong shedding relative to placebo among those with higher baseline viral RNA levels. Furthermore, Lambda appeared to prolong shedding relative to placebo among those who were seronegative at baseline. Although these subgroup analyses were exploratory and should only be considered hypothesis generating, our findings are not consistent with a possible benefit of Lambda among individuals with higher viral loads (or seronegative individuals early in the course of disease).

These data are in contrast to reports of benefits of Type I IFN in hospitalized COVID-19 patients. Subcutaneous IFN- $\beta$ along with ribavirin and lopinavir/ritonavir was associated with shortened duration of symptoms and viral shedding in hospitalized patients in Hong Kong ${ }^{14}$, and a reduction in mortality when given with hydroxychloroquine and lopinavir/ritonavir or atazanavir-ritonavir in Iran ${ }^{17}$. Furthermore, a randomized clinical trial in England also suggest clinical benefits of inhaled IFN- $\beta$ for COVID-19 15 . Although both type I and type III IFN activate the same dominant JAK-STAT signaling pathway ${ }^{6}$, inhibit SARSCoV-2 in vitro ${ }^{10,11}$, and have receptors on respiratory epithelia ${ }^{18}$, in vivo activity and efficacy may differ ${ }^{6}$. A recent report found that inborn errors of Type I IFN immunity, including autosomal recessive IFNAR1 deficiency, were enriched in patients with lifethreatening COVID-19 pneumonia ${ }^{35}$. Furthermore, patients with COVID-19 pneumonia were also more likely to have neutralizing auto-antibodies against type I, but not type III, IFNs ${ }^{36}$. These data suggest the possibility that type I IFN administration may be more beneficial than type III IFN in preventing adverse outcomes of SARS-CoV-2 infection.

The majority (62.5\%) of participants in our study were Latinx, reflecting the high burden of COVID-19 among the Latinx community in our surrounding communities ${ }^{37}$. Minority populations are disproportionately affected by COVID-19, with higher rates of infection and deaths observed due to a multitude of socioeconomic and demographic factors ${ }^{38,39}$. Attention has recently been called to the relative absence of the most affected minorities in treatment trials ${ }^{40,41}$, and we prioritized recruitment efforts to the Latinx community in our study.

The study did have a few limitations. We recruited both symptomatic and asymptomatic patients. Asymptomatic patients contributed less to secondary outcomes since they presented with lower viral RNA levels and could not contribute to analyses of symptom alleviation. However, these patients represented $<10 \%$ of the enrolled cohort. Additionally, despite a reported median duration of symptoms prior to randomization of only 5 days, $40 \%$ of participants were already seropositive at enrollment. A recently published outpatient study of a monoclonal antibody cocktail with similar study design (REGN-COV2) found similar rates of baseline SARS-CoV-2 IgG seropositivity $(45 \%)^{42}$. These data suggest that enrolling COVID-19 outpatients early in the course of disease, before they develop an antibody response, may be challenging. Nonetheless, we found no suggestion of benefit of Lambda in seronegative individuals. We do not provide data on viral measures indicative of active replication (e.g., tissue culture infectious dose (TCID) $)_{50}$ assays), although samples with CT values $>24$ have been reported to have very low levels of being tissue culture positive ${ }^{43}$. Finally, the median time to cessation in the placebo arm was shorter than assumed in our sample size calculations, potentially due to less severe disease in this population. However, our original sample size estimates based on the number of events and median time to event were conservative; a 
shorter time to cessation, keeping all other assumptions the same, increases the power to detect differences between groups.

In conclusion, a single dose of subcutaneous Peginterferon Lambda-1a, while safe, neither reduced time to cessation of viral shedding nor symptom duration in outpatients with uncomplicated COVID-19 in this large, Phase 2, single-center study. Further investigation into the therapeutic utility of subcutaneous Peginterferon Lambda-1a for COVID-19 in patients with severe illness (NCT04343976) or as a prophylactic treatment (NCT04344600) are underway.

\section{Methods}

Trial design and oversight. We conducted a Phase 2, single-blind, randomized placebo-controlled trial to evaluate the efficacy of Lambda in reducing the duration of viral shedding in outpatients. The trial was conducted within the Stanford Health Care System. Adults aged 18-65 years with an FDA emergency use authorized reverse transcription-polymerase chain reaction (RT-PCR) positive for SARS-CoV-2 within $72 \mathrm{~h}$ from swab to the time of enrollment were eligible for participation in this study. Both symptomatic and asymptomatic patients were included given reports finding that both CT values and presence of infectious virus were similar in samples from asymptomatic and symptomatic persons ${ }^{44}$. Symptomatic individuals were eligible given the presence of mild to moderate symptoms without signs of respiratory distress. Asymptomatic individuals were eligible if infection was initial diagnosis of SARS-CoV-2 infection. Exclusion criteria included current or imminent hospitalization, respiratory rate $>20$ breaths per minute, room air oxygen saturation $<94 \%$, pregnancy or breastfeeding, history of decompensated liver disease, recent use of interferons, antibiotics, anticoagulants or other investigational and/or immunomodulatory agents for treatment of COVID-19, and prespecified lab abnormalities. Full eligibility criteria are provided in the study protocol. The protocol was amended on June 16, 2020 after 54 participants were enrolled but before results were available to include adults up to 75 years of age and eliminate exclusion criteria for low white blood cell and lymphocyte count. The trial was registered at ClinicalTrials.gov (NCT04331899). The study was performed as an investigator initiated clinical trial with the FDA (IND 419217), and approved by the Institutional Review Board of Stanford University.

Recruitment and enrollment. Participants were recruited with flyers, online advertising, and phone calls to Stanford patients with positive SARS-CoV-2 RTPCR. Recruitment materials and phone calls were provided in multiple languages, including English and Spanish. After confirming eligibility and providing informed consent in the patient's primary language, participants underwent a standardized history and physical exam, and completed bloodwork. If inclusion criteria were met, participants were randomly assigned to Lambda or placebo using a 1:1 REDCAP-based computer-generated randomization scheme that stratified by age ( $\geq 50$ and $<50$ years old) and sex. A password-protected electronic spreadsheet containing the randomization allocation, along with the code used to generate the allocation and seed used in the random number generation, was stored on secure servers at Stanford.

Study drug administration. Phase 2 studies established the optimal dose for virologic suppression and minimizing treatment-related adverse events (mainly aminotransferase and/or bilirubin elevations) for hepatitis $\mathrm{C}$ at $180 \mathrm{mcg}$ given subcutaneously ${ }^{23}$. This dose is also currently being used in hepatitis D trials, and was provided by Eiger BioPharmaceuticals for use in this study. Those assigned to Lambda received a single $180 \mathrm{mcg}$ subcutaneous injection of study drug $(0.45 \mathrm{ml}$ volume), and those assigned to placebo received a $0.45 \mathrm{ml}$ subcutaneous injection of saline (prepared by the Stanford Investigational Pharmacy). A single injection was chosen given Lambda's prolonged half-life (mean $T_{1 / 2} 63-117 \mathrm{~h}^{29}$ ) and ease of single-dose administration. The study medication/placebo syringe was dispensed by the Stanford Investigational Pharmacist and administered by a study nurse. Lambda and placebo syringes were identically labeled but differed in the appearance of the needle hub. Since the nurse administering the medication might see syringe differences, the study was not strictly "double-blind" even though all participants and investigators were blinded to treatment arm. Participants were monitored for adverse events for thirty minutes after injection.

Participant follow up. Participants completed a daily symptom questionnaire using REDCap Cloud version 1.5. Participants also provided in-home measurements of temperature and oxygen saturation using study-provided devices. Inperson follow-up visits were conducted at Day $1,3,5,7,10,14,21$, and 28, with assessment of symptoms and vitals, and collection of oropharyngeal swabs (FLOQ Swabs; Copan Diagnostics). Peripheral blood was also collected at Day 5 and 14 to assess for safety events.

Laboratory procedures. Laboratory measurements were performed by trained study personnel using point-of-care CLIA-waived devices or in the Stanford Health
Care Clinical Laboratory. Oropharyngeal swabs were tested for SARS-CoV-2 in the Stanford Clinical Virology Laboratory using an emergency use authorized, laboratory-developed, RT-PCR ${ }^{45-47}$. Centers for Disease Control and Prevention guidelines identify oropharyngeal swabs as acceptable upper respiratory specimens to test for the presence of SARS-CoV-2 RNA ${ }^{26}$, and detection of SARS-CoV-2 RNA swabs using oropharyngeal swabs was analytically validated in the Stanford virology laboratory.

IgG antibody titers against the SARS-CoV-2 spike receptor-binding domain (RBD) were assessed at enrollment by enzyme-linked immunosorbent assay $\left(\right.$ ELISA) ${ }^{27}$. Briefly, $\mathrm{His}_{6}$-tagged SARS-CoV-2 RBD construct (gift from F. Krammer, Icahn School of Medicine at Mount Sinai) was transiently transfected into Expi293F cells (Thermo Fisher Scientific). After transfection, cell culture supernatants were collected by centrifugation for protein purification. Protein purity was assessed by SDS-PAGE. Round-bottom 96-well plates (Immunolon 2HB; Thermo Scientific) were coated with $50 \mu \mathrm{l}$ of RBD at $2 \mu \mathrm{g} \mathrm{ml}^{-1}$ in PBS. Heatinactivated serum samples at enrollment were diluted 5-fold starting at 1:50 and IgG antibody titers against RBD determined by ELISA. Absorbance was measured at $450 \mathrm{~nm}$ (SPECTRAmax 250, Molecular Devices). Samples were considered seropositive against RBD if their absorbance value was greater than the mean plus four standard deviation (SD) of all negative controls $(n=130)$.

Data and safety monitoring. Adverse events were assessed and graded for severity according to standardized criteria ${ }^{20}$. A Data and Safety Monitoring Board (DSMB) was established and conducted an interim analysis to review clinical trial progress, integrity, and safety data.

Study outcomes. The primary pre-specified outcome was time to first of two consecutive negative oropharyngeal tests for SARS-CoV-2 by RT-PCR. Secondary outcomes included: (1) Time to alleviation of all symptoms, defined as time until the first day when no symptoms were reported; (2) SARS-CoV-2 oropharyngeal viral RNA levels over time; (3) SARS-CoV-2 oropharyngeal viral RNA area under the curve (AUC); and (4) Incidence of emergency department visits or hospitalizations within 28 days of initiation of treatment. Adverse events (AEs) and serious AEs (SAEs) were the primary safety endpoints. For secondary outcomes utilizing oropharyngeal viral RNA levels, we used the following conversion formula from cycle threshold values to copies/ml PBS:

$$
10 \wedge\left(-0.288^{*} \mathrm{Ct}+11.007\right) \times 120
$$

Exploratory outcomes included: (1) time until sustained resolution of symptoms, defined as the first day when no symptoms were reported for the duration of the study; (2) Progression of disease, defined as admission to the emergency department, hospitalization, or worsening cough or shortness of breath defined as an increase in severity of two points or more on a five-point scale. Primary, secondary, and exploratory outcomes were pre-specified in our statistical analysis plan and finalized prior to database lock.

Statistical analysis. Analyses were performed according to assigned randomization arm (intent-to-treat). All models were covariate-adjusted for the randomization strata of age group and sex. Unless otherwise noted, all tests were two-sided and conducted at the 0.05 level of significance. Analyses were performed in $\mathrm{R}$ version $4.0 .2^{48}$.

Primary analysis. Time until shedding cessation was compared between arms using a Cox proportional hazards model covariate-adjusted for age and sex, with the final hypothesis test conducted at the alpha $=0.04999$ level of significance to allow for an interim analysis. The hazard ratio for shedding cessation was estimated, along with its 95\% confidence interval. Participants who dropped out prior to having two consecutive negative tests were censored at the time of their last positive test or on Day 1 if no positive tests were observed. The proportional hazards assumption was verified by examining the Schoenfeld residuals. Efron's approximation was used to handle ties in the Cox proportional hazards model.

Secondary analysis. Time until resolution of symptoms was compared using a Cox proportional hazards model. Hazard ratios and 95\% confidence intervals were reported. Change in viral load during follow-up was compared using a linear mixed-effects model with random intercepts for participant. The AUC of viral load was compared using linear regression. Multiple imputation using chained equations was used to impute missing viral load data prior to area under the curve calculation. Five data sets were imputed, and imputed values calculated using non missing viral load on each of the 7 sample collection days, treatment arm, age, sex, and whether or not a participant was hospitalized. Model estimates were pooled across the five imputed datasets by computing the total variance over the repeated analyses.

Estimates for change in viral load and viral load AUC for lambda compared to placebo and corresponding 95\% confidence intervals for the linear models were reported. The incidence of hospitalizations and emergency department visits was estimated for each arm, with $95 \%$ confidence intervals. AEs were compared by arm using the Chi-squared test and Fisher's exact test for SAEs. 
A sensitivity analysis was performed for the primary endpoint using only symptomatic patients at baseline. Because two participants, after randomization, inadvertently were injected with the incorrect syringe, we also conducted an astreated analysis according to treatment actually received.

A statistical interaction term between treatment arm and symptomatic status at baseline was added to the Cox proportional hazards model adjusted for age group and sex to test whether symptomatic status was an effect modifier of the relationship between treatment and time to shedding cessation. The main effect of each potential effect modifier was also included in the model. Additional effect modifiers specified a priori were (1) having a CT value $<30$ (vs. $\geq 30$ ) on baseline oropharyngeal swab, (2) IgG seropositivity at baseline, and (3) number of risk factors or predictors for severe disease present at baseline (temperature $\geq 99.5$, cough, or shortness of breath present at randomization [symptoms count as a single risk factor], age $\geq 60$, male sex, Black race, Hispanic ethnicity, body mass index $\geq 30$, and lab values of baseline lymphocyte counts $<1000$ and baseline ALT 294). Effect modification was considered significant if the $p$ value for interaction was $<0.05$.

Post hoc analyses were conducted to test for differences in both median duration of symptoms pre-randomization and baseline $\log _{10}$ viral load between seronegative and seropositive participants. The Kruskal-Wallis rank sum was used to test for differences in symptom duration while a two-sample $t$-test was used to test for differences in $\log _{10}$ viral load.

Sample size determination. Assuming 1:1 randomization and the use of a twosided $\log$ rank test at the alpha $=0.04999$ level of significance for the final analysis, we anticipated the occurrence of 79 shedding cessation events, which provided $80 \%$ power to detect a hazard ratio of 2.03 . We additionally assumed median time to shedding cessation of 14 days in the control arm and 7 days in the treatment arm, a 2-month accrual period, a 2-week follow-up period after randomization of the last patient, and 10\% drop out in the control arm. This enabled an interim analysis conducted at alpha $=0.00001$ to assess overwhelming efficacy after $50 \%$ of participants completed $24 \mathrm{~h}$ of follow-up. We estimated that the total sample size required to achieve 79 events was 120 (60 participants per arm)

Ethical approval. The study was registered as an investigator initiated clinical trial with the FDA (IND 419217), and approved by the Institutional Review Board of Stanford University. Written informed consent was provided by all study participants.

Reporting summary. Further information on research design is available in the Nature Research Reporting Summary linked to this article.

\section{Data availability}

The datasets generated and analyzed during the current study are permanently available in the Stanford Digital Repository at the following URL: https://purl.stanford.edu/ hc972ys 6733

Source data underlying all figures including those in supplementary information are provided at the above URL.

Received: 22 December 2020; Accepted: 3 March 2021;

Published online: 30 March 2021

\section{References}

1. COVID-19 Dashboard by the Center for Systems Science and Engineering (CSSE) at Johns Hopkins University (JHU) (Johns Hopkins University, 2021). https://coronavirus.jhu.edu/map.html.

2. Baud, D. et al. Real estimates of mortality following COVID-19 infection Lancet Infect. Dis. 20, 773 (2020).

3. Park, A. \& Iwasaki, A. Type I and Type III interferons-induction, signaling, evasion, and application to combat COVID-19. Cell Host Microbe 27, 870-878 (2020).

4. Kotenko, S. V. IFN-lambdas. Curr. Opin. Immunol. 23, 583-590 (2011).

5. Zhou, Z. et al. Type III interferon (IFN) induces a type I IFN-like response in a restricted subset of cells through signaling pathways involving both the JakSTAT pathway and the mitogen-activated protein kinases. J. Virol. 81, 7749-7758 (2007).

6. Lazear, H. M., Schoggins, J. W. \& Diamond, M. S. Shared and distinct functions of Type I and Type III interferons. Immunity 50, 907-923 (2019).

7. Yuen, C. K. et al. SARS-CoV-2 nsp13, nsp14, nsp15 and orf6 function as potent interferon antagonists. Emerg. Microbes Infect. 9, 1418-1428 (2020).

8. $\mathrm{Chu}, \mathrm{H}$. et al. Comparative replication and immune activation profiles of SARS-CoV-2 and SARS-CoV in human lungs: an ex vivo study with implications for the pathogenesis of COVID-19. Clin. Infect. Dis. 71, 1400-1409 (2020).
9. Arunachalam, P. S. et al. Systems biological assessment of immunity to mild versus severe COVID-19 infection in humans. Science 369, 1210-1220 (2020).

10. Hadjadj, J. et al. Impaired type I interferon activity and inflammatory responses in severe COVID-19 patients. Science 369, 718-724 (2020).

11. Felgenhauer, U. et al. Inhibition of SARS-CoV-2 by type I and type III interferons. J. Biol. Chem. 295, 13958-13964 (2020).

12. Dinnon, K. H. et al. A mouse-adapted model of SARS-CoV-2 to test COVID19 countermeasures. Nature 586, 560-566 (2020).

13. Zhou, Q. et al. Interferon-alpha2b treatment for COVID-19. Front. Immunol. 11, 1061 (2020).

14. Hung, I. F. et al. Triple combination of interferon beta- $1 \mathrm{~b}$, lopinavir-ritonavir, and ribavirin in the treatment of patients admitted to hospital with COVID19: an open-label, randomised, phase 2 trial. Lancet 395, 1695-1704 (2020).

15. Monk, P. D. et al. Safety and efficacy of inhaled nebulised interferon beta-1a (SNG001) for treatment of SARS-CoV-2 infection: a randomised, doubleblind, placebo-controlled, phase 2 trial. Lancet Respir. Med. 9, 196-206 (2021).

16. Dastan, F. et al. Subcutaneous administration of interferon beta-1a for COVID-19: a non-controlled prospective trial. Int. Immunopharmacol. 85, 106688 (2020).

17. Davoudi-Monfared, E. et al. A Randomized Clinical Trial of the efficacy and safety of interferon beta-1a in treatment of severe COVID-19. Antimicrob. Agents Chemother. 64, e01061 (2020).

18. Mordstein, M. et al. Lambda interferon renders epithelial cells of the respiratory and gastrointestinal tracts resistant to viral infections. J. Virol. $\mathbf{8 4}$ 5670-5677 (2010).

19. Sommereyns, C., Paul, S., Staeheli, P. \& Michiels, T. IFN-lambda (IFNlambda) is expressed in a tissue-dependent fashion and primarily acts on epithelial cells in vivo. PLoS Pathog. 4, e1000017 (2008).

20. Pagliaccetti, N. E., Chu, E. N., Bolen, C. R., Kleinstein, S. H. \& Robek, M. D. Lambda and alpha interferons inhibit hepatitis B virus replication through a common molecular mechanism but with different in vivo activities. Virology 401, 197-206 (2010).

21. Robek, M. D., Boyd, B. S. \& Chisari, F. V. Lambda interferon inhibits hepatitis B and C virus replication. J. Virol. 79, 3851-3854 (2005).

22. Davidson, $\mathrm{S}$. et al. IFNlambda is a potent anti-influenza therapeutic without the inflammatory side effects of IFNalpha treatment. EMBO Mol. Med. 8, 1099-1112 (2016)

23. Muir, A. J. et al. A randomized phase $2 \mathrm{~b}$ study of peginterferon lambda-1a for the treatment of chronic HCV infection. J. Hepatol. 61, 1238-1246 (2014).

24. O'Brien, T. R. et al. Weak induction of interferon expression by severe acute respiratory syndrome Coronavirus 2 Supports Clinical Trials of Interferonlambda to treat early Coronavirus Disease 2019. Clin. Infect. Dis. 71, 1410-1412 (2020)

25. Prokunina-Olsson, L. et al. COVID-19 and emerging viral infections: the case for interferon lambda. J. Exp. Med. 217, e20200653 (2020).

26. Centers for Disease Control and Prevention. CDC 2019-nCoV Real-Time RTPCR Diagnostic Panel instructions for use (effective February 4, 2020). https:// www.cdc.gov/coronavirus/2019-ncov/lab/guidelines-clinical-specimens.html.

27. Chakraborty, S. et al. Proinflammatory IgG Fc structures in patients with severe COVID-19. Nat. Immunol. 22, 67-73 (2020)

28. Chan, H. L. Y. et al. Peginterferon lambda for the treatment of $\mathrm{HBeAg}$ positive chronic hepatitis B: A randomized phase $2 \mathrm{~b}$ study (LIRA-B). J. Hepatol. 64, 1011-1019 (2016).

29. Eiger_BioPharmaceuticals. Investigators Brochure version 3 11, May 2018 (Eiger_BioPharmaceuticals, 2018).

30. Nair, A. B. \& Jacob, S. A simple practice guide for dose conversion between animals and human. J. Basic Clin. Pharm. 7, 27-31 (2016).

31. Major, J. et al. Type I and III interferons disrupt lung epithelial repair during recovery from viral infection. Science 369, 712-717 (2020).

32. Broggi, A. et al. Type III interferons disrupt the lung epithelial barrier upon viral recognition. Science 369, 706-712 (2020).

33. Egli, A. et al. IL-28B is a key regulator of B- and T-cell vaccine responses against influenza. PLoS Pathog. 10, e1004556 (2014).

34. Feld, J. J. et al. Peginterferon-lambda for the treatment of COVID-19 in outpatients. medRxiv, 2020.2011.2009.20228098 (2020).

35. Zhang, Q. et al. Inborn errors of type I IFN immunity in patients with lifethreatening COVID-19. Science 370, eabd4570 (2020).

36. Bastard, P. et al. Autoantibodies against type I IFNs in patients with lifethreatening COVID-19. Science 370, eabd4585 (2020).

37. Center, C.o.S.C.E.O. Santa Clara County COVID-19 Demographics Dashboard, Vol. 2020 (Center, C.o.S.C.E.O)

38. Price-Haywood, E. G., Burton, J., Fort, D. \& Seoane, L. Hospitalization and mortality among Black patients and White patients with Covid-19. N. Engl. J. Med. 382, 2534-2543 (2020).

39. Stokes, E. K. et al. Coronavirus Disease 2019 Case Surveillance- United States, January 22-May 30, 2020. Morb. Mortal. Wkly Rep. 69, 759-765 (2020).

40. Chastain, D. B. et al. Racial disproportionality in Covid Clinical Trials. $N$. Engl. J. Med. 383, e59 (2020). 
41. Chowkwanyun, M. \& Reed, A. L. Jr. Racial health disparities and Covid-19caution and context. N. Engl. J. Med 383, 201-203 (2020).

42. Weinreich, D. M. et al. REGN-COV2, a neutralizing antibody cocktail, in outpatients with Covid-19. N. Engl. J. Med. 384, 238-251 (2020).

43. Bullard, J. et al. Predicting infectious SARS-CoV-2 from diagnostic samples. Clin. Infect. Dis. ciaa638 https://doi.org/10.1093/cid/ciaa638 (2020). Epub ahead of print.

44. Singanayagam, A. et al. Duration of infectiousness and correlation with RTPCR cycle threshold values in cases of COVID-19, England, January to May 2020. Eur. Surveill. 25, 2001483 (2020).

45. Hogan, C. A., Sahoo, M. K. \& Pinsky, B. A. Sample pooling as a strategy to detect community transmission of SARS-CoV-2. JAMA 323, 1967-1969 (2020).

46. Hogan, C. A. et al. Comparison of the Panther Fusion and a laboratorydeveloped test targeting the envelope gene for detection of SARS-CoV-2. J. Clin. Virol. 127, 104383 (2020).

47. Corman, V. M. et al. Detection of 2019 novel coronavirus (2019-nCoV) by real-time RT-PCR. Eur. Surveill. 25, 2000045 (2020).

48. Team, R.C. R: A Language and Environment for Statistical Computing (R Foundation for Statistical Computing, 2019).

\section{Acknowledgements}

We thank all study participants who participated in this study, and the study team for their tireless work. We also thank our colleagues at Stanford University Occupational Health and at San Mateo Medical Center for participant referrals. Financial support from Stanford's Innovative Medicines Accelerator and operational support from Stanford ChEM-H is acknowledged. The Stanford REDCap platform (http://redcap.stanford.edu) is developed and operated by Stanford Medicine Research IT team. The REDCap platform services at Stanford are subsidized by (a) Stanford School of Medicine Research Office, and (b) the National Center for Research Resources and the National Center for Advancing Translational Sciences, National Institutes of Health, through grant UL1 TR001085. The study was funded by anonymous donors to Stanford University, and Lambda provided by Eiger BioPharmaceuticals. Additional support was provided from NIH/NIAID (U01 AI150741-01S1 to P.J. and T.W.). The funders had n.o role in data collection and analysis or the decision to publish.

\section{Author contributions}

P.J., J.R.A., J.P., and U.S. designed the study and wrote the study protocol. C.L., H.H., J.P., V.B. developed study data instruments. C.H., I.C. provided study drug. P.J., J.R.A., H.B., K.B.J., S.K., C.R.d.V., O.Q., K.F., D.W., J.N., K.E., C.B., T.W., B.A.P., J.P., and U.S. collected data. C.H., I.C., Y.M., J.G., A.B., C.K. provided input on study procedures and data analysis. H.H., N.P., V.B., and M.D. prepared the statistical analysis plan and analyzed the data. All authors participated in data interpretation. K.B.J. and P.J. wrote the first draft and writing the manuscript and agreed on the decision to publish. There were no confidentiality agreements between the sponsors and authors.

\section{Competing interests}

C.H. and I.C. are scientists at Eiger BioPharmaceuticals, Inc., which provided the Interferon Lambda used for this study. J.G. serves on the board of Eiger BioPharmaceuticals, Inc. C.H. and I.C. own stock and options of Eiger BioPharmaceuticals, Inc. J.G. has an equity interest in Eiger BioPharmaceuticals, Inc. J.G. and I.C. are inventors on a pending patent application relating to the use of interferon lambda for coronavirus. Eiger BioPharmaceuticals played no role in study design, conduct of the study, or analysis of the data. All other authors declare no competing interests.

\section{Additional information}

Supplementary information The online version contains supplementary material available at https://doi.org/10.1038/s41467-021-22177-1.

Correspondence and requests for materials should be addressed to P.J. or U.S.

Peer review information Nature Communications thanks the anonymous reviewers for their contribution to the peer review of this work. Peer reviewer reports are available.

Reprints and permission information is available at http://www.nature.com/reprints

Publisher's note Springer Nature remains neutral with regard to jurisdictional claims in published maps and institutional affiliations.

\begin{abstract}
(c) (1) Open Access This article is licensed under a Creative Commons Attribution 4.0 International License, which permits use, sharing, adaptation, distribution and reproduction in any medium or format, as long as you give appropriate credit to the original author(s) and the source, provide a link to the Creative Commons license, and indicate if changes were made. The images or other third party material in this article are included in the article's Creative Commons license, unless indicated otherwise in a credit line to the material. If material is not included in the article's Creative Commons license and your intended use is not permitted by statutory regulation or exceeds the permitted use, you will need to obtain permission directly from the copyright holder. To view a copy of this license, visit http://creativecommons.org/ licenses/by/4.0/.
\end{abstract}

() The Author(s) 2021 